\title{
Ein Prosit der Gesundheit
}

\author{
In Bier stecken viele dermatologisch nutzbare Ingredienzien. Prof. Dr. Dr. Johannes Ring und \\ seine Kollegen von der TU München haben sich die Mühe gemacht, die bisherigen Veröffent- \\ lichungen zum Thema zu sichten und zu bewerten.
}

Es sind vor allem die Polyphenole im Bier, die viele der Gesundheit dienliche Eigenschaften haben: Sie wirken antibakteriell, antientzündlich, verhindern Gefäßneubildung, Osteoporose und schützen vor Krebs. Hopfen enthält etwa Kaempferol, Quercetin, Tyrosyl, Ferulasäure, Humulone und Lupulone, die dem Bier den charakteristischen bitteren Geschmack verleihen.

\section{Hinweise für antikanzerogene Wirkung}

Kaempferol ist für Onkologen interessant, weil epidemiologischen Studien darauf hinweisen, dass es das kardiovaskuläre sowie das Krebsrisiko verringert. Zumindest im Tiermodell konnte belegt werden, dass es der UVB-induzierten Karzinogenese in der Haut entgegenwirkt. Das geschieht, indem die Cox-2-Proteinsynthese in bestimmten epidermalen Zellen unterdrückt wird. Laut Ring et al. linderte die topische Applikation von Kaempferol nach leichtgradigen Verbrennungen die Folgen der Einwanderung von Entzündungszellen in die geschädigten Hautareale.

Eine eher antioxidative Wirkungen entfaltet die Ferulasäure, ein Vorläufermolekül des Vanillins. Es gibt bereits viele In-vitroDaten zur Wirksamkeit dieser Substanz, was Forscher einen therapeutischen Effekt bei neurodegenerativen und kardiovaskulären Erkrankungen sowie Diabetes und Krebs vermuten lässt. Auch häufen sich Hinweise, dass die Substanz einen photoprotektiven Effekt hat und die Wundheilung beschleunigt. Aber entsprechende Effekte in Untersuchungen mit Patienten sind noch nicht hieb- und stichfest belegt. Das gilt auch für die krebshemmende Wirkung. Oral verabreichte Ferulasäure verhinderte die Entstehung von Hauttumoren, die experimentell bei Mäusen in der Kontrollgruppe ohne die Substanz durch topisch aufgetragenes Dimethylanthracen ausgelöst wurden. Und in zwei von drei prospektiven Studien über zehn Jahre konnte zwar keine Assoziation zwischen Biertrinken und der Entstehung eines Basalzellkarzinoms festgestellt werden, in der dritten, aktuelleren Studie wurde jedoch eine Reduktion des Risikos beobachtet. Doch der Beleg einer Assoziation zwischen Biertrinken und der Entstehung bzw. Verhinderung von Hautkrebs steht noch aus.

Das Polyphenol Xanthohumol findet man nur im Hopfen. Es ist in der Lage, die Interleukin-12-Synthese zu blockieren. Im Tiermodell für chronische allergische Kontaktdermatitis, durch Oxazolon ausgelöst, wurde die Hautreaktion signifikant durch Applikation von Xanthohumol reduziert. Die Wissenschaftler spekulieren, dass Erwachsene mit einem atopischen Ekzem von

\section{Bittere Wahrheiten \\ Für die pharmakologische Wirkung des Bieres ist vor allem der Hopfen verantwortlich. Er verleiht dem Gerstensaft sein ausgeprägtes Aroma und seine typische Bitter- keit. Zum Brauen werden aus- schließlich die Dolden der weib- lichen Pflanzen verwendet, die nicht befruchtet sein dürfen. Die Kultivierung männlicher Pflanzen ist daher in den Anbaugebieten streng verboten. Das größte geschlossene Hopfenanbaugebiet der Welt ist die Hallertau in Oberbayern.}

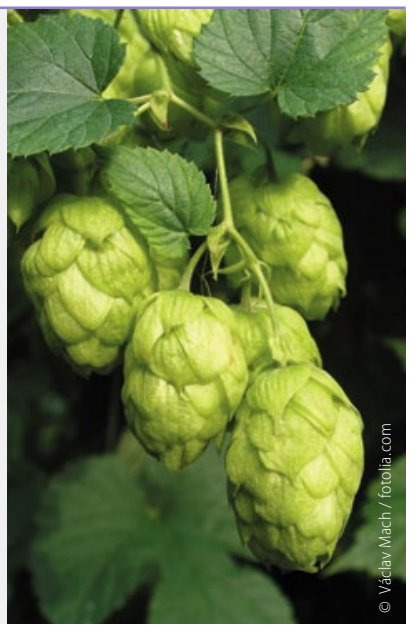

einem moderaten Bierkonsum profitieren würden, wobei noch entsprechende Studien fehlen.

\section{Einfluss auf das Haarwuchstum}

Die für das Bierbrauen verwendete Gerste enthält wie Äpfel auch Procyanidin, das auf das Haarwachstum wirkt. In der placebokontrollierten japanischen Studie wurde 0,7\%-iges Procyanidin aus Apfelextrakt bei Männern auf die Glatze aufgetragen. Je länger behandelt wurde, umso dichter wurde der Haarwuchs - ohne Nebenwirkungen. Auch wenn es viele kosmetische Produkte, auf dem Markt gebe, die Hopfenextrakte enthielten, fehlten bisher klinische Studien, die den Effekt auf Hautalterung und Haarwuchs belegten, so die Wissenschaftler.

Die Dermatologen lassen bei der Erforschung der Bieringredienzien nicht locker. Vielversprechend sind dabei die immer effektiveren Applikationssysteme für das Auftragen etwa von Xanthohumol, Quercetin oder Ferulasäure. Auch wenn wohl das Biertrinken selbst nicht zur Therapie von Hauterkrankungen empfohlen werden kann, so sind die bisherigen Forschungsergebnisse zu den Inhaltsstoffen im Zusammenhang mit einer möglichen therapeutischen Anwendung doch ermutigend.

Peter Leiner

Chen W et al. J Eur Acad Dermatol Venereol 2013 Jun 27 [Epub ahead of print] 